\title{
EFEITO CAUSAL DO PROGRAMA MAIS MÉDICOS SOBRE O ATENDIMENTO DO PROGRAMA MÃE CORUJA A PARTIR DOS MICRODADOS
}

\author{
J.R.S. de ANDRADE ${ }^{1}$, R. G. de ARRUDA ${ }^{2}$ e P.H.P.M. VAZ \\ ${ }^{1}$ Universidade Federal de Pernambuco, Centro de Ciências Sociais Aplicada, Discente \\ do Programa de Pós-Graduação em Gestão e Economia da Saúde \\ 2 Faculdade Nova Roma, Docente do curso de Economia \\ ${ }^{3}$ Universidade Federal de Pernambuco, Centro de Ciências Sociais Aplicada, Docente \\ do Departamento de Economia \\ E-mail para contato: joseanerafaelasa@gmail.com
}

\begin{abstract}
RESUMO - O Programa Mãe Coruja Pernambucana (PMCP) visa o cuidado da gestante e da criança até 5 anos de idade através da articulação com a rede de saúde e outros serviços existente no município. Trata-se de um estudo longitudinal de avaliação do efeito de uma politica com abordagem quantitativa dos dados em painel com informações das mulheres e crianças dos 105 municípios beneficiados pelo programa. Tendo como objetivo principal avaliar que fatores influenciam a efetividade do Mãe Coruja sobre as taxas de mortalidade infantil, através da análise dos microdados disponíveis no monitoramento mensal do SISmãecoruja, no periodo de 2013 a 2016. E como objetivos específicos traçar o perfil de gestantes e crianças assistidas pelo programa e mensurar se houve maior efetividade do PMCP sobre as taxas de mortalidade infantil nas cidades que aderiram ao Programa Mais Médicos (PMM) elou possuem elevada cobertura da Estratégia de Saúde da Família (ESF). O método econométrico Logit foi utilizado através do software STATA, onde a variável dependente é óbito infantil, e a variável PMM indica se a mãe cadastrada no PMCP pariu em um periodo posterior à implantação do PMM no município de residência. Sendo -0.3408412 o coeficiente, que é estatisticamente significante ( $p$ valor menor que 0,05). Logo, podemos dizer que a adesão ao PMM nos municípios onde existe o PMCP reduz a probabilidade de óbito infantil, tudo o mais mantido constante.
\end{abstract}

PALAVRAS-CHAVE: Avaliação de Política Pública; Economia da Saúde; Programa Mãe Coruja Pernambucana; Programa Mais Médico do Brasil

ABSTRACT - The Mãe Coruja Pernambucana program (PMCP) aims to care for pregnant women and children up to 5 years old through articulation with the health network and other services that exist in the city. This is a longitudinal impact assessment study of a policy with a quantitative panel data approach with information from women and children of the 105 municipalities benefiting from the program. The main objective of this study was to evaluate the influence of the Mãe Coruja Pernambucana program effectiveness on infant mortality rates, through the analysis of the microdata available in the monthly monitoring of SISmãecoruja, from 2013 to 2016. As specific objectives, the study intends to establish the profile of pregnant women and children that are assisted by the program and to measure if there was a greater effectiveness of PMCP over infant mortality rates in cities that have adhered to Mais Médicos Program and/or have a high 
coverage of Estratégia de Saúde da Familia. The econometric method Logit was used through the STATA software, where the dependent variable is infant death, and the $P M M B$ variable indicates if the mother registered in the PMCP gave birth in a subsequent period to the PMMB implantation in the municipality of residence. The coefficient of 0.3408412 is statistically significant (value of " $p$ " is less than 0.05). Therefore, we conclude that adherence to the PMMB in the municipalities where PMCP exists reduces the probability of infant death, if all the rest is maintainedconstant. KEYWORDS: Public Policy Evaluation; Health Economics; Mãe Coruja Pernambucana Program; Mais Médicos do Brasil Program.

\section{INTRODUÇÃO}

No atual cenário, onde os recursos estão cada vez mais escassos e a sociedade já vivencia um debate e compreende que é necessário avaliar com cautela antes de se investir em Políticas Públicas e analisar quais os benefícios reais que a implantação de um novo programa possa trazer de retorno no médio e/ou longo prazo para a sociedade (NOVAES, 2000).

As altas taxas de mortalidade infantil são um grave problema de saúde pública e se configura como uma violação dos direitos humanos, atingindo desigualmente as regiões brasileiras com maior prevalência nas classes com menor poder aquisitivo. Com intuito de contribuir com a melhoria da atenção à saúde da criança e da mulher no estado de Pernambuco, a implantação do Programa Mãe Coruja Pernambucana (PMCP), se deu de forma transversal e estratégica naqueles que apresentavam os piores indicadores, estando presente em 105 dos 185 municípios do Estado (PERNAMBUCO, 2016).

Enquanto Programa Mais Médicos (PMM) surge na tentativa de reduzir o déficit de médicos na Atenção Básica ( $\mathrm{AB}$ ), ampliar o acesso aos serviços de saúde, reduzir tempo de espera e garantir assistência à saúde especialmente aos grupos mais vulneráveis, além de promover melhoria na infraestrutura das Unidades Básicas e formar profissionais preparados para atuar neste nível de atenção (BRASIL,2015).

Esta pesquisa justifica-se pela necessidade de aprofundar estudos anteriores como Cabral et. al (2016), visando entender melhor os canais de efetividade da política, utilizando os microdados para aumentar o poder de inferência estatística. E tem como objetivo mensurar estatisticamente por meio de métodos econométricos o quanto houve de mudança no indicador de mortalidade infantil em Pernambuco após a implantação do Programa Mais Médicos nos municípios que já possuíam p Programa Mãe Coruja.

Desenvolver esse estudo tem sido um trabalho desafiador e ambicioso pois é a primeira vez que alguém utiliza os microdados - disponíveis no monitoramento mensal através do Sistema de Informação "SIS Mãe Coruja", para esse tipo de análise, possibilitando um aprofundamento quanto a heterogeneidade dos efeitos do programa.

\section{MATERIAIS E MÉTODOS}

Trata-se de um estudo longitudinal de avaliação do efeito de uma política, com abordagem quantitativa dos microdados em painel. Sendo o instrumento de coleta as Planilhas de Excel, no período de janeiro de 2013 a dezembro de 2016, geradas pelo SISmãecoruja e disponibilizadas pela SES para os pesquisadores mediante aprovação prévia no CEP/UFPE.

O método econométrico Logit foi utilizado através do software STATA, tendo como estratégia de identificação do Grupo Tratado as gestantes cadastradas no PMCP que pariram após a chegada do PMM em seu município. 


\section{RESULTADOS E DISCUSSÃO}

Tabela 1 - Efeito do Programa Mais Médicos na redução da mortalidade infantil

\begin{tabular}{|c|c|c|c|c|c|}
\hline $\begin{array}{l}\text { ÓBITO } \\
\text { INFANTIL }\end{array}$ & $\begin{array}{l}\text { COEFICIE } \\
\text { N. }\end{array}$ & $\begin{array}{c}\text { ERRO } \\
\text { PADRÃO }\end{array}$ & $\mathbf{Z}$ & $\mathbf{P}>|\mathbf{Z}|$ & $\begin{array}{l}\text { INTERVALO DE } \\
\text { CONFIANÇA } 95 \%\end{array}$ \\
\hline PMM & -0.3408412 & 0.1537972 & -2.22 & 0.027 & $-0.6422782 \quad-0.0394041$ \\
\hline IDADE & -0.0007463 & 0.0061221 & -0.12 & 0.903 & $-0.0127453 \quad 0.0112527$ \\
\hline RISCO & 0.8336054 & 0.1017032 & 8.20 & 0.000 & $0.6342709 \quad 1.03294$ \\
\hline PARTO & & & -1.90 & 0.057 & -0.3154353 \\
\hline CONSULTAS & -0.2759446 & 0.0156298 & -17.66 & 0.000 & -0.3065785 \\
\hline
\end{tabular}

Nota: Foram introduzidos efeitos fixos de município e de período

Na Tabela 1, a variável dependente é óbito infantil, enquanto a variável PMM indica que a mãe cadastrada no Programa Mãe Coruja (PMC) pariu posteriormente à implantação do Programa Mais Médicos (PMM) pelo município de residência, onde ela teve acesso ao pré-natal. Sendo o valor do coeficiente -0.3408412 , que é estatisticamente significante.

Tabela 2 - Efeito da implantação do Programa Mais Médicos sobre a probabilidade do óbito infantil

\begin{tabular}{|c|c|c|c|c|c|c|}
\hline ÓBITO & LOGIT & LOGIT & LOGIT & LOGIT & LOGIT & LOGIT \\
\hline INFANTIL & 1 & 2 & 3 & 4 & 5 & 6 \\
\hline PMM & $-0.2319^{*}$ & $-0.2382 *$ & $-0.2316^{*}$ & $-0.2282 *$ & $-0.1527 * * *$ & $-0.3408^{*}$ \\
\hline IDADE & & 0.0003 & -0.0058 & -0.0046 & -0.0001 & -0.0007 \\
\hline RISCO & & & $0.7376^{*}$ & $0.7623 *$ & $0.8539^{*}$ & $0.8336^{*}$ \\
\hline PARTO & & & & $-0.224 * *$ & $-0.1475 * *$ & $-0.1554 * *$ \\
\hline CONSULTAS & & & & & $-0.2618 *$ & $-0.2759 *$ \\
\hline
\end{tabular}

A Tabela 2 mostra que quando se controla o coeficiente aumentando progressivamente as variáveis, o coeficiente se manteve negativo e significante, o que indicando que o programa surtiu efeito na redução da mortalidade infantil.

\section{CONCLUSÃO}

Logo, podemos dizer que a implantação do Programa Mais Médicos nos municípios onde já existe o Programa Mãe Coruja reduz a probabilidade de óbito infantil, tudo o mais mantido constante.

\section{REFERÊNCIA}

BRASIL, Ministério da Saúde. Secretaria de Gestão do Trabalho e da Educação na Saúde. Programa mais médicos - dois anos: mais saúde para os brasileiros. Brasília, 2015. 
NOVAES, H. M. D. Avaliação de programas, serviços e tecnologias em saúde. Revista de Saúde Pública, Ed.5. 2000. Disponível em: $<$ http://www.revistas.usp.br/rsp $>$ Acesso: $14 / 11 / 2016$.

PERNAMBUCO, Sistema de Informação e Gestão da Assistência Social de Pernambuco (SES). Programa Mãe Coruja Pernambucana. Recife, 2016. Disponível em: $<$ https://www.sigas.pe.gov.br/pagina/programa-me-coruja-pernambucana $>$. Acesso em: 25/05/2017.

\section{AGRADECIMENTOS}

Agradecemos a Coordenação Estadual do Programa Mãe Coruja e do Programa Mais Médicos pela contribuição para o desenvolvimento da pesquisa; e ao Ministério da Saúde pelo financiamento do Programa de Pós-Graduação em Gestão e Economia da Saúde (PPGGES). 